\title{
L'„Italia Pontificia“ e le vicende degli ordini religioso-militari nella Penisola
}

\author{
Kristjan Toomaspoeg
}

Il convegno dedito al centesimo anniversario dell'impresa editoriale dell'Italia Pontificia ospita due relazioni che riguardano gli ordini religioso-militari medievali: quella del prof. Rudolf Hiestand, incentrata sulla storia generale degli ordini in Terra Santa e in Europa e sui destini dei loro archivi ${ }^{1}$, e la presente, finalizzata a illustrare gli aspetti della presenza dei tre principali ordini militari, ovvero quelli dei Giovanniti, Templari e Teutonici, nel preciso ambito della Penisola Italiana.

Come è noto, la Curia pontificia medievale ha emesso numerosi atti relativi agli ordini militari nella Penisola, il che spiega il ruolo rilevante avuto da queste fonti nel lavoro di Paul Fridolin Kehr e dei suoi collaboratori. Da questo punto di vista, si distingue soprattutto la sezione dell'Italia Pontificia che riguarda l'isola di Malta: essa si basa sulla documentazione giovannita e templare, rintracciata negli antichi archivi centrali dell'Ordine di Malta ${ }^{2}$. Però, delle unità archivistiche sull'argomento sono state individuate anche in altri ambiti geografici studiati dagli storici tedeschi.

Ad esempio, nella Biblioteca Comunale di Palermo, Kehr consultò le copie degli atti dei Templari e degli Ospitalieri di Sicilia realizzate nel Seicento dallo storiografo regio Antonino Amico ${ }^{3}$. Il manoscritto di Amico contiene molte

1 Si veda in questo volume, pp. 615-672.

2 Paul Fridolin KeHR: Papsturkunden in Malta, in: NGG phil.-hist. Klasse (1899) pp. 369-409 (rist. in Paul Fridolin KeHR: Papsturkunden in Italien. Reiseberichte zur Italia Pontifica, vol. 2, Città del Vaticano 1977 [Acta Romanorum pontificum 2], pp. 89-130). Nell'introduzione di questa opera, basata sulle ricerche condotte da Luigi Schiaparelli, Kehr spiega anche le ragioni della decisione di includere Malta nel progetto dell'Italia Pontificia, motivata più che altro dalla necessità di confrontare gli atti degli antichi archivi dell'Ordine di Malta nell'isola, in sé allora già abbastanza noti, con i documenti, spesso sconosciuti, conservati tra i resti dell'archivio priorale giovannita di Messina, in Sicilia. Nel volume sono pubblicate 53 lettere apostoliche che riguardano sia i Giovanniti che i Templari e sono presentati i regesti di molte decine di altri documenti.

3 Si tratta del manoscritto numero Qq H 12 della Biblioteca Comunale di Palermo, redatto fra il 1625 e il 1629, dal titolo: Diplomata, litterae etc. ad sacram domum militum S. Joannis Hyerosolymitani et militum Templariorum pertinentia. Per Antonino Amico e il suo manoscritto, si veda Raffaele Starrabba: Scritti inediti o rari di Antonino Amico e documenti relativi al medesimo, Palermo 1891; Giuseppina Pecorella: I Templari nei manoscritti di Antonino Amico, Palermo 1921; Kristjan Toomaspoeg: 
lettere apostoliche del XII secolo tra cui alcune erano sconosciute prima della loro presentazione nell'Italia Pontificia ${ }^{4}$. Altri atti sulla storia dei Templari o degli Ospitalieri si trovano nei fondi delle Chiese capitolari o delle congregazioni religiose moderne in tutto il territorio italiano.

Al tempo stesso, alcuni cartulari degli ordini militari non censiti all'interno dell'Italia Pontificia, come quelli dei Teutonici a Palermo ${ }^{5}$ e a Padova ${ }^{6}$,

Templari e Ospitalieri nella Sicilia Medievale, Bari 2003 (Gran Priorato di Napoli e Sicilia del Sovrano Militare Ordine di Malta, Melitensia 11), pp. 26-36.

4 Paul Fridolin KeHR: Papsturkunden in Sizilien, in: NGG, phil.-hist. Klasse (1899) pp. 283-337 (rist. in KeHR, Papsturkunden in Italien [cit. nota 2] pp. 33-87). In Sicilia, le ricerche archivistiche furono condotte da Luigi Schiaparelli e Paul Fridolin Kehr con il contributo del giovane Karl Andreas Kehr. Il volume si compone in gran parte da documenti del manoscritto di Antonino Amico: così gli atti pubblicati con numeri 2, pp. $311-312$, 3, p. 313 (abbreviato), 4, pp. 313-314, 5, p. 314 (abbr.), 7, p. 315, 11, p. 319 (abbr.), 12, pp. 319-320, 13, p. 320 (abbr.), 14, p. 321, 15, pp. $321-322$, 16, pp. $322-333,18$, pp. $324-325,20$, pp. $326-327,22$, p. 328 (abbr.), 23, p. 328 (abbr.), 24, pp. 328-329, 25, p. 329 (abbr.), 27, p. 330 (abbr.), 33, pp. 333-334. Il manoscritto Qq H 12 era indubbiamente la principale fonte inedita degli studiosi dell'Italia Pontificia nell'isola, e troviamo nel volume anche una brevissima presentazione del manoscritto e del suo autore (KeHR: Papsturkunden in Sizilien [cit. questa nota] pp. 294-295), completata e resa più esaustiva in KeHR: Papsturkunden in Malta (cit. nota 2) p. 370.

5 Palermo, Archivio di Stato, Tabulario della Magione. A questa raccolta di 801 pergamene si aggiungono molte copie degli atti medievali, custodite nei fondi Commenda della Magione e Miscellanea Archivistica II. In tutto, troviamo tra la documentazione ,teutonica' dell'Archivio di Stato 20 lettere apostoliche. Per gli studiosi dell'Italia Pontificia, il fondo del Tabulario della Magione era „ohne Papsturkunden“ (KeHR: Papsturkunden in Sizilien [cit. nota 4] p. 287).

6 Nell'Archivio di Stato di Padova, nel fondo Corporazioni Religiose Soppresse, Gesuiti, sono state individuate 422 pergamene provenienti dall'antico archivio della provincia di ,Lombardia' dell'Ordine Teutonico, tra le quali 17 sono delle lettere apostoliche medievali. Si veda la breve presentazione del fondo in Kristjan Toomaspoeg: La fondazione della provincia di "Lombardia“ dell'Ordine dei Cavalieri Teutonici (secoli XIII-XIV), in: Sacra Militia 3 (2003) pp. 111-159. Gli studiosi dell'Italia Pontificia non ebbero grande interesse per questo fondo archivistico: esso si compone di manoscritti moderni e le pergamena medievali sono state rilegate all'interno di alcuni volumi del fondo, cosicché bisognava prima di tutto individuare la loro collocazione. Questo lavoro è stato svolto solo molti decenni più tardi da Carlo Polizzi nella sua Tesi di laurea rimasta inedita: Comune, signoria ezzeliniana e Chiesa di Padova nel secolo XIII attraverso le carte dei frati alemanni. Con due appendici: 1. Edizione di 57 documenti inediti (1232-1463), 2. Regesto e regesto-estratto di 316 documenti inediti (1219-1399), tesi di laurea, Università degli Studi di Padova, 1981-1982. Si veda anche Carlo Polizzi: Proprietà, feudi e livelli di molini e canali della Padova comunale. Pubblicato a cura dell'autore (art. 9 L 2-2-1939 n. 374), Padova 1989 e Sante Bortolami: Acque, mulini e folloni nella formazione del paesaggio urbano medievale (secoli XI-XIV): l'esempio di Padova, in: Paesaggi urbani dell'Italia padana nei secoli VIII-XIV, Bologna 1988, pp. 277-330, che si servono entrambi dei documenti dell'archivio teutonico. 
conservano ancora delle lettere apostoliche inedite; inoltre si continuano a scoprire documenti nuovi anche tra i numerosi transunti e copie realizzati in epoca moderna ${ }^{7}$ : questo fatto induce a continuare le ricerche intraprese a loro tempo da Kehr e i suoi collaboratori e a completare il quadro delle relazioni intercorse tra il papato e gli ordini militari, anche se sembra ancora lontana la possibilità della creazione di un corpus diplomatico esaustivo degli ordini militari in Italia ${ }^{8}$.

In Italia esiste una tradizione storiografica di studi sulla storia degli ordini religioso-militari che risale al XVI secolo ${ }^{9}$. Durante gli ultimi decenni, la ricerca ha fatto notevoli progressi, anche se, a tutt'oggi, poche sono le monografie che trattano l'argomento per l'intera Penisola, ovvero al di fuori dei confini regionali ${ }^{10}$. Di recente, i lavori preliminari alla stesura del „Dictionnaire des

7 Nell'ambito delle ricerche sull'Ordine Teutonico, Hubert Houben ha individuato a Brindisi, Graz, Napoli, Vienna e altrove numerose copie dei documenti sulla provincia pugliese dei Teutonici, compensando in questo modo la perdita degli atti originali avvenuta nel 1943 con la distruzione del deposito dell'Archivio di Stato di Napoli a San Paolo Belsito: si vedano in particolare Hubert Houben: Zur Geschichte der Deutschordensballei Apulien. Abschriften und Regesten verlorener Urkunden aus Neapel in Graz und Wien, in: MIÖG 107 (1999) pp. 50-110; ID.: Friedrich II., der Deutsche Orden und die Burgen im Königreich Sizilien. Eine unbekannte Urkunde Honorius' III von 1223, in: DA 56 (2000) pp. 585-591; ID.: Neuentdeckte Papsturkunden für den Deutschen Orden (1219-1261) im Staatsarchiv Neapel, in: QFIAB 83 (2003) pp. 41-82. Nell'ambito delle ricerche templari e giovannite, si veda ad esempio Antonella Pellettieri: Militia Christi in Basilicata. Storia e diffusione degli Ordini religioso-cavallereschi (secc. XII-XIX), Potenza 2005 (Quaderni di Leukanikà 4) che si basa sulle copie manoscritte napoletane.

8 Un tentativo di questo genere, che avrebbe potuto seguire l'esempio di Rudolf Hiestand: Papsturkunden für Templer und Johanniter. Archivberichte und Texte, Göttingen 1972 (AAG, phil.-hist. Klasse, 3. Folge 77, Vorarbeiten zum Oriens Pontificius 1) non è mai stato effettuato. Di qualche limitato aiuto è Gaettano Lamattina: Regesta pontificum romanorum. Erga Templarios (1139-1313), Roma 1984 (manoscritto in libera consultazione nella Biblioteca Apostolica Vaticana) e tutte le edizioni dei fondi archivistici ,centrali' degli ordini religioso-militari che comprendono anche la documentazione emessa dai pontefici.

9 Le problematiche della storiografia degli ordini religioso-militari in Italia sono state affrontate nel corso del recente seminario internazionale di studi: Le Crociate: nuove prospettive di ricerca (Roma, Consiglio Nazionale delle Ricerche, 16-17 marzo 2006). Si aspetta la (prossima) pubblicazione degli atti di questo seminario per aggiungere delle informazioni ad un argomento ancora poco studiato.

10 L'unica monografia scientifica ed esaustiva (con dovute critiche) sulla storia di un ordine religioso-militare nell'ambito di tutta la Penisola è Fulvio Bramato: Storia dell'Ordine dei Templari in Italia, Roma 1991-1994. Per il resto possediamo dei saggi su alcuni aspetti specifici degli ordini in Italia, come ad esempio Anthony LutTrell: Templari e Ospitalieri in Italia, in: La chiesa di San Bevignate a Perugia, a cura di Mario RoncetTi/ Pietro Scarpellini/Francesco Tommasi, Perugia 1987, pp. 19-26, e gli articoli contenuti in Anthony Luttrell: The Hospitallers of Rhodes and their Mediterranean 
ordres militaires", un ambizioso progetto europeo che si concretizzerà con un'edizione prevista per il 2009, hanno reso possibile l'incontro e la collaborazione di tutti gli studiosi che lavorano sul territorio italiano ${ }^{11}$.

La mia comunicazione propone una riflessione sui rapporti diretti fra la Sede Apostolica e le rappresentanze in Italia dei tre maggiori ordini religiosomilitari. In questa occasione, presenterò la rilettura di una serie di fonti pontificie, che provengono da quattro diversi secoli, per riflettere sul ruolo svolto dal papato nell'insediamento degli Ospitalieri e dei Templari nella Penisola nel XII secolo; sulla relazione tra la Sede Apostolica e i Teutonici, i Templari e gli Ospitalieri nel XIII secolo e sul quadro storico successivo al Processo ai Templari e all'indebolimento della Sede Apostolica nel XIV secolo. Infine, concluderò portando alcuni esempi della trasformazione dei possedimenti dei Teutonici in benefici ecclesiastici soggetti al potere pontificio nel XV secolo.

\section{Il papato e il primo insediamento italiano degli ordini religioso-militari nel XII secolo}

Non esiste alcun dubbio sul fatto che la creazione degli ordini religioso-militari nella prima metà del XII secolo sia stata voluta e favorita dalla Sede Apostolica, unica autorità predisposta a dare corpo all'ideologia di san Bernardo di Chiaravalle, malgrado la sua evidente contraddizione con lo spirito della riforma ecclesiastica, dal momento che riunì in sé due categorie sociali, i monaci e $\mathrm{i}$ cavalieri, capovolgendo l'ordine della società ${ }^{12}$.

Il papa divenne, almeno in teoria, l'unica autorità ecclesiastica e politica alla quale gli ordini dovevano obbedienza e rimase sempre il loro principale interlocutore. Il ruolo e l'influenza esercitati dal papato risultano evidenti anche guardando alle vicende storiche degli ordini religioso-militari nella Penisola Italiana. Dalle fonti emesse dalla Sede Apostolica si evince un'attività abbastanza intensa di preparazione finalizzata all'insediamento degli ordini in Italia, intrapresa già prima della creazione del concetto di militia Christi, quando gli Ospitalieri di San Giovanni di Gerusalemme, così come altre congregazioni

World, Aldershot 1992, o sulle vicende degli ordini in singole aree geografiche della Penisola.

11 Dictionnaire des ordres militaires au Moyen Âge (Paris 2009), frutto della collaborazione degli storici di quasi tutti i paesi europei, compresi, per la parte italiana, gli studiosi come Pier Carlo Begotti/Elena Bellomo/Renato Bordone/Giampaolo Cagnin/Fulvio Delle Donne/Hubert Houben/Andrea Lercari/Francesco Tommasi.

12 Si veda il riassunto in Alain Demurger: Vie et mort de l'ordre du Temple, Paris 1993 (edizione aggiornata) e ID.: Chevaliers du Christ. Les ordres religieux-militaires au Moyen Âge $\left(\mathrm{XI}^{\mathrm{e}}-\mathrm{XVI}^{\mathrm{e}}\right.$ siècle), Paris 2002. 
religioso-assistenziali della Terra Santa, furono oggetto di particolare attenzione da parte del papato per il loro ruolo di difesa e assistenza dei pellegrini.

Questo interesse divenne manifesto con l'emanazione, a Benevento, di due lettere apostoliche di Pasquale II del 15 febbraio del 1113: Latorem presencium, con cui si ordinava ai prelati e ai fedeli di collaborare per la raccolta delle elemosine degli Ospitalieri in Occidente ${ }^{13}$, e Pie postulatio voluntatis ${ }^{14}$. Questo secondo documento, come è noto, dichiarava gli Ospitalieri sotto la speciale protezione del pontefice e confermava all'institutor ac prepositus dell'Ospedale gerosolimitano, Geraudo, il possesso di una serie di ospedali, ubicati a SaintGilles, Asti, Pisa, Bari, Otranto, Taranto e Messina. La lettera, emessa non molto tempo dopo l'avvenuta emancipazione dell'Ospedale dalla tutela dei Benedettini e l'inizio della sua espansione, serve ancora oggi agli storici per datare la presenza dei Giovanniti in tutte le località che vi sono elencate, quindi in territorio provenzale e italiano. Però, mentre il documento papale afferma con chiarezza che l'Ospedale gerosolimitano era già presente in queste località, un esame approfondito delle altre fonti disponibili ci obbliga ad ammettere che la lettera non era una conferma per il possesso di questi beni, bensì un atto programmatico, con cui si approvava la linea che gli Ospitalieri intendevano seguire nel loro insediamento.

Infatti, la stessa Casa di Saint-Gilles non è documentata prima del $1121^{15}$, benché i Giovanniti fossero già presenti in Provenza (a Tolosa e Béziers) intorno al $1110^{16}$; ad Asti la chiesa dell'Ordine viene menzionata solo nel $1169^{17}$; a Bari e Otranto, esso non ebbe mai beni rilevanti ${ }^{18}$; mentre le Case di Taranto e Messina sono state fondate vari decenni più tardi ${ }^{19}$. La lettera Pie postulatio voluntatis non diede quindi risultati immediati e le strade seguite dai Giovanniti nel loro insediamento furono spesso diverse da quelle indicate nel documento,

13 Cartulaire général de l'Ordre des Hospitaliers de S. Jean de Jérusalem (1100-1310), a cura di Joseph Marie Antoine Delaville Le Roulx, Paris 1894-1904, vol. 1 p. 30 n. 31.

14 Ibid. vol. 1 pp. $29-30$ n. 30 .

15 Ibid. vol. 1 pp. $45-46$ n. 54.

16 Si veda ivi pp. 2-24 nn. 3, 7, 15, 17, 18, 23.

17 Renato Bordone/Donatella GNeTtr: Distribuzione geografica delle „domus“ gerosolimitane in Piemonte e situazione patrimoniale del gran priorato di Lombardia (commenda di Asti), in: „Gentilhuomini christiani e religiosi cavalieri“: nove secoli dell'Ordine di Malta in Piemonte, a cura di Tomaso Ricardi di Netro/Luisa Clotilde Gentile, Milano 2000, pp. 142-145.

18 Mariarosaria Salerno: Gli ospedalieri di San Giovanni di Gerusalemme nel Mezzogiorno d'Italia (secc. XII-XV), Bari 2001 (Gran Priorato di Napoli e Sicilia del Sovrano Militare Ordine di Malta, Melitensia 8)

19 Per Messina, si veda Kristjan Toomaspoeg : Templari e Ospitalieri nella Sicilia Medievale, Bari 2003 (Gran Priorato di Napoli e Sicilia del Sovrano Militare Ordine di Malta, Melitensia 11), pp. 54sg. 
tuttavia era manifesta la volontà di Pasquale II di collocare la congregazione lungo le vie di transito dall'Occidente verso la Terra Santa.

Le circostanze mutarono con la creazione dell'Ordine del Tempio e la progressiva trasformazione dell'Ospedale in un ordine religioso-militare. I nuovi ordini furono protetti e favoriti dal papato nei loro possedimenti in Terra Santa, mentre il loro stanziamento nell'attuale territorio italiano si inserisce all'interno di un contesto politico molto particolare, venutosi a creare con la doppia elezione pontificia del 14 febbraio 1130 dei due papi rivali, Anacleto II e Innocenzo II.

Anacleto esercitò il suo potere per lo più a Roma e nell'Italia meridionale, dove fece incoronare il suo alleato Ruggero II come primo re di Sicilia. Innocenzo, influente nel resto della Penisola, fu sostenuto da Bernardo di Chiaravalle e dai suoi protetti, i Cistercensi e i Templari. Di conseguenza, nell'Italia meridionale i Templari furono praticamente assenti fino alla pacificazione avvenuta tra Ruggero II e Innocenzo II nel $1140^{20}$, mentre nell'Italia centrale dove erano favoriti da Innocenzo, essi riuscirono a fondare alcune delle loro Case principali, come quelle di Pisa, Lucca e Siena ${ }^{21}$.

Tra il 1130 e il 1150, i Templari e gli Ospitalieri, grazie al sostegno di Innocenzo II e dei suoi successori, crearono le basi della loro presenza nel territorio italiano, ricevendo ovunque donazioni da parte di privati e autorità pubbliche. Il papato, senza concedere beni immobiliari agli ordini, favorì il loro insediamento tramite l'emanazione di privilegi che ricordavano gli esoneri e la protezione speciale dei quali entrambi godevano e anche tramite una serie di lettere indirizzate ai prelati di determinate aree geografiche, destinate a facilitare le attività dei frati quali la raccolta delle donazioni e dell'elemosina. Ad esempio, la lettera Milites Templi Jherosolimitani di Lucio II, del 5 giugno $1144^{22}$, ribadita nel novembre del 1145 o 1146 da Eugenio III $^{23}$, chiede agli ecclesiastici siciliani

20 Si veda Kristjan Toomaspoeg: L'insediamento dei grandi ordini militari cavallereschi in Sicilia, 1145-1220, in: La presenza dei cavalieri di San Giovanni in Sicilia. Convegno internazionale, Palermo, Palazzo dei Normanni, 17 giugno 2000 - Messina, Palazzo Zanca, 18 giugno 2000, Roma 2001 (Fondazione Melitense „Donna Maria Marullo di Condojanni“, Collana di Studi 1/1), pp. 41-51, e iD.: Le patrimoine des grands ordres militaires en Sicile, 1145-1492, in: Mélanges de l'École française de Rome, Moyen Âge 113/1 (2001) pp. 313-341.

21 Anthony LutTRell: The Hospitallers of Rhodes between Tuscany and Jerusalem, 1310 1431, in: Revue Mabillon 64 (1992) pp. 117-138 (rist. in: Anthony LutTrell:, The Hospitallers State of Rhodes and its Western Provinces, 1306-1462, Aldershot 1999 [Variorum Collected Studies Series 655], n. XVII).

22 Regesto in Lamattina: Regesta (cit. nota 8) p. 11. Questa lettera non è identica all'altra con gli stessi incipit e data, più nota, indirizzata a tutti i prelati della Chiesa: Hiestand: Papsturkunden (cit. nota 8) pp. 215sg. n. 9.

23 Regesto in KeHr: Papsturkunden in Sizilien (cit. nota 4) p. 63 n. 3; ed. Cartulaire général de l'Ordre du Temple 1119?-1150. Recueil des Chartes et des Bulles relatives à 
di assistere i Templari con donativi e sussidi; così la lettera Milites Templi Domini di Adriano IV, del 7 settembre $1157^{24}$, tratta della conservazione dei beni dei Templari. A queste lettere apostoliche, destinate a contesti specifici, se ne aggiungono molte altre di contenuto generico, trascritte e confermate dalle autorità locali: ad esempio, la lettera Cum fratribus Hospitalis di Alessandro III, del 2 marzo 1174, 75 o $76^{25}$, nota solo grazie ad una copia messinese, riassunta dal Kehr in „Papsturkunden in Sizilien“, che ricorda a tutti i prelati della Chiesa alcuni dei principali privilegi degli Ospitalieri.

A partire dal quarto decennio del XII secolo, si forma quindi un corpus di lettere apostoliche piuttosto consistente riguardante i diritti dei Templari e degli Ospitalieri in Italia e attestante la preoccupazione del papato per la conservazione del patrimonio locale degli ordini, considerato come una garanzia di assistenza ai pellegrini e agli altri viaggiatori diretti in Terra Santa e, sempre più spesso, anche come un vero e proprio sostegno logistico della Terra Santa.

Questo specifico aspetto ricorre con frequenza nella corrispondenza pontificia di Eugenio III (1145-53) e Alessandro III (1159-1181), l'uno in origine Cistercense, l'altro molto favorevole ai Templari. Al termine del pontificato di Alessandro III, i due ordini maggiori si erano stabiliti in Toscana, Emilia e Romagna, Lombardia, Marche, Basilicata, Campania, Puglia e Sicilia e presto riuscirono a stanziarsi nelle restanti aree geografiche, come la Liguria, il Friuli e la Calabria.

A questa epoca si attesta la presenza degli ordini anche nel Patrimonio di San Pietro, in particolare a Roma ${ }^{26}$, dove i Templari si insediarono probabilmente subito dopo la morte di Anacleto II, avvenuta nel gennaio 1137: nel 1138, Bernardo di Chiaravalle visitò la loro nova domus in Urbe. Tuttavia, come abbiamo detto, il sostegno del papato agli ordini non consisté in donazioni d'immobili: è noto infatti che sia la chiesa templare di Santa Maria all'Aventino che quella giovannita di San Basilio al Foro di Augusto $^{27}$ furono acquisiti da

l'Ordre du Temple, a cura del marchese D’Albon, Paris 1913, p. 383 n. XIV; Lamattina: Regesta (cit. nota 8) p. 12 (datazione errata).

24 KeHR: Papsturkunden in Sizilien (cit. nota 4) pp. 63sg. n. 4.

25 Ibid. p. 69 n. 11.

26 Si veda L'ordine templare nel Lazio meridionale: atti del convegno, Sabaudia, 21 ottobre 2000, a cura di Clemente Ciammaruconi, Casamari 2003 (Ministero per i beni e le attività culturali. Direzione generale per i beni librari e gli istituti culturali. Biblioteca statale del monumento nazionale di Casamari); Anne Gilmour-Bryson: The Trial of the Templars in the Papal State and Abruzzi, Città del Vaticano 1982; Annibale Ilari: Il Granpriorato giovannita di Roma: ricerche storiche ed ipotesi, Bari 1998 (Gran Priorato di Napoli e Sicilia del Sovrano Militare Ordine di Malta, Melitensia 4); Giulio Silvestrelli: Le chiese e i feudi dell'Ordine dei Templari e dell'Ordine di San Giovanni di Gerusalemme nella regione romana, in: Rendiconti della Reale Accademia dei Lincei 5, 26 (1917) pp. 490-520.

27 Sulle chiese romane degli ordini militari si veda Mariano Armellini: Le chiese di Roma dal secolo IV al XIX, Roma ${ }^{2} 1891$, pp. 146sg., 501-503, 587sg. 
terzi, anche se è lecito pensare ad un intervento confermativo della Sede Apostolica. Nel Lazio, i possedimenti degli ordini rimasero per molti decenni poco significativi, e anche altrove furono rari gli esempi di città pontificie, come Ascoli Piceno nelle Marche $^{28}$, dotate di importanti insediamenti templari o ospitalieri.

Il caso del Patrimonio di San Pietro è indicativo dell'atteggiamento tenuto dalla Sede Apostolica rispetto allo stanziamento degli ordini religioso-militari in Italia nel XII secolo: l'arrivo e le attività degli ordini furono facilitati e privilegiati in tutti i modi da quasi tutti i pontefici, ma senza però intaccare il proprio patrimonio.

\section{Il papato e gli ordini religioso-militari nel XIII secolo}

La presenza degli ordini religioso-militari, come di tutte le congregazioni della Terra Santa, fu rafforzata nella Penisola Italiana dopo la sconfitta del 1187 e la perdita di Gerusalemme. Si tratta di un fenomeno che accompagnava la ricerca di una base logistica europea per la Terra Santa, descritto, per quanto riguarda il Mezzogiorno d'Italia, da Geneviève Bresc-Bautier ${ }^{29}$. Nell'arco dei tre primi decenni del XIII secolo, il Tempio, l'Ospedale e il nuovo ordine dei frati dell'Ospedale di Santa Maria dei Teutonici ultimarono la creazione della rete dei loro possedimenti in territorio italiano, trasformando spesso i loro primi nuclei, dediti all'assistenza dei pellegrini e degli infermi, in centri di produzione agricola. La potenza del patrimonio degli ordini fece sì che le loro sedi nei principali centri politici della Penisola giocassero anche il ruolo di vere e proprie rappresentanze diplomatiche.

Nel XIII secolo gli Ospitalieri di San Giovanni stabilirono i loro principali priorati italiani a Venezia, Pisa, Roma, Capua, Barletta e Messina, mantenendo, delle sedi indicate nella lettera di Pasquale II nel 1113, solo quelle di Pisa e Messina. I possedimenti dell'Ospedale coprirono quasi tutto l'odierno territorio italiano divenendo quindi l'ordine religioso-militare più presente nella Peniso$\mathrm{la}^{30}$.

28 Anthony Lutrrell: The Hospitaller Priory of Venice, in: Militia Sacra. Gli ordini militari tra Europa e Terrasanta, a cura di Enzo Coli/Maria De Marco/Francesco Tommasi, Perugia 1994, pp. 101-143; Gabriele Petromilli: I Templari della Marca Centrale, Ancona 1984.

29 Geneviève Bresc-Bautier: Les possessions des églises de Terre Sainte en Italie du Sud (Pouille, Calabre, Sicile), in: Roberto il Guiscardo e il suo tempo. Relazioni e comunicazioni nelle prime Giornate normanno-sveve (Bari, maggio 1973), Roma 1975 (Fonti e studi del Corpus membranarum italicarum 11), pp. 13-39.

30 Francesco Tommasi: L'ordinamento geografico-amministrativo dell'Ospedale Gerosolimitano di San Giovanni in Italia (secc. XII-XIV), in: Religiones Militares. Contributi 
I Templari furono particolarmente presenti in alcune regioni, come la Toscana e le Marche, e possederono beni rilevanti in altre; ma, per una serie di circostanze politiche e diplomatiche, acquistarono relativamente pochi beni nel Regno di Sicilia ${ }^{31}$.

I Teutonici seguirono strade completamente diverse nel loro stanziamento: da un lato, perché questo avvenne quasi un secolo dopo rispetto ai Templari e Ospitalieri, dall'altro, per un indiscusso legame con la politica imperiale tedesca d'Enrico VI e Federico II e con la Sede Apostolica d'Innocenzo III e, soprattutto, di Onorio III. Nella prima metà del XIII secolo i Teutonici crearono i loro baliati in Sicilia, nel Mezzogiorno continentale e in „Lombardia“, ovvero nel territorio veneto, friulano e emiliano, avevano beni anche in Toscana e nel Lazio, senza dimenticare la loro presenza nel Tirolo meridionale, nei territori di lingua tedesca ${ }^{32}$.

L'insediamento dell'Ordine Teutonico fornisce un altro esempio interessante della politica pontificia rispetto agli ordini religioso-militari. Innocenzo III, sebbene avesse riconosciuto l'Ordine, ebbe per molto tempo un atteggiamento cauto riguardo la sua presenza italiana (che, all'epoca, consisteva solo nei possedimenti di Puglia e Sicilia). Così, il 2 agosto del 1198, in una lettera indirizzata all'arcivescovo palermitano ${ }^{33}$, il papa risolse il problema dell'occupazione manu militari del monastero cistercense della Santissima Trinità di Palermo da parte dell'esercito di Enrico VI e dei Teutonici in modo ambiguo, consentendo ai Teutonici di rimanere nel monastero, a patto che si comportassero „senza eccessi“, ovvero senza eccedere troppo nella loro adesione al partito imperiale.

alla storia degli Ordini religioso-militari nel medioevo, a cura di Anthony LutTrelL/ Francesco Tomması, Città di Castello 2008 (Biblioteca di “Militia sacra” 2), pp. 61 130.

31 Bramato: Storia (cit. nota 10); Toomaspoeg: Templari (cit. nota 19); Luttrell: Templari e Ospitalieri (cit. n. 10); Elena Bellomo: The Templar Order in North-West Italy (1142-c. 1330), Leiden/Boston 2008 (The Medieval Mediterranean 72).

32 L'Ordine Teutonico nel Mediterraneo. Atti del Convegno internazionale di studio Torre Alemanna (Cerignola)-Mesagne-Lecce 16-18 ottobre 2003, a cura di Hubert Houben, Galatina 2004 (Acta Theutonica 1), offre un quadro aggiornato della presenza dei Teutonici in Italia, per quanto riguarda le aree di lingua tedesca della Penisola, si veda Der Deutsche Orden in Tirol. Die Ballei an der Etsch und im Gebirge, a cura di Heinz Noflatscher, Marburg 1991 (Quellen und Studien zur Geschichte des Deutschen Ordens 43).

33 Antonio Mongitore: Monumenta historica sacrae domus mansionis SS. Trinitatis militaris ordinis Theutonicorum urbis Panormi et magni ejus praeceptoris. Origo, privilegia, immunitates, praeceptores, commendatarii, ecclesiae suffraganae, proventus, aliaque memorabilia ejusdem sacrae domus recensentur et illustrantur. Auctore sacrae theologiae doctore D. Antonio Mongitore, Palermo 1721, p. 15. Si veda anche, per il quadro storico, Friedrich BaEthGen: Die Regentschaft Papst Innozenz III. im Königreich Sizilien, Heidelberg 1914.. 
Durante gli anni dell'alleanza fra Federico II e Onorio III l'Ordine Teutonico, sotto la guida di Ermanno di Salza, visse una delle fasi di massima espansione giuridica, politica ed economica. Senza entrare nel merito dei numerosi privilegi concessi ai Teutonici da Onorio III, noti attraverso molti studi, vorrei fare alcune osservazioni sulla loro concreta applicazione in territorio italiano.

Innanzitutto, si assiste ad alcune concessioni di beni e redditi a favore dell'Ordine Teutonico effettuate da Federico II con l'approvazione del papato, sia perché il sovrano doveva obbedienza al papa per il Regno di Sicilia, sia per il fatto che si trattava di concessioni fatte nell'ambito dei preparativi delle spedizioni in Terra Santa. Così, nel dicembre del $1216^{34}$ e il 24 giugno del $1217^{35}$, Federico II diede ai Teutonici dei redditi fiscali rispettivamente nei porti di Brindisi e Messina, ma solo quelli di Messina furono confermati da Onorio III il 21 marzo 1218, con la lettera Iustis petentium desideriiis ${ }^{36}$. In questo ultimo caso, il reddito di 200 once d'oro doveva servire ai frati in attesa di partire per la Terra Santa per procurarsi mantelli per l'inverno, mentre la concessione di Brindisi, ignorata dal papa, fu fatta in cambio di alcuni non precisati beni in Germania che i Teutonici consegnarono a Federico II. Nel 1219, il re, senza volerlo, violò i diritti giurisdizionali della Sede Apostolica quando diede ai Teutonici alcuni terreni a Medicina, vicino Bologna: il conflitto fu risolto con una lettera di scuse di Federico a Onorio III e la tacita approvazione del papato ${ }^{37}$.

In Italia il legame tra i Teutonici, il papato e l'autorità reale e poi imperiale di Federico II si concretizzò inizialmente nell'ambito dei preparativi della crociata, ma presto il sostegno di Onorio III trovò altre motivazioni. Una lettera del papa dell'11 dicembre del 1223, recentemente scoperta e pubblicata da

34 Jean Louis Alphonse Huillard-BrÉHolles: Historia diplomatica Friderici secundi, Paris 1859-1861, vol. 1, pp. 488-490; Codex diplomaticus ordinis sanctae Mariae Theutonicorum, a cura di Johann Heinrich Hennes, Mainz 1845, vol. 2, p. 5, Gaston von Pettenegg: Die Urkunden des Deutsch-Ordens Zentralarchives zu Wien, vol. 1 (1170-1809), Praga/Leipzig 1887, p. 9 n. 22, regesto in RI 5/1 p. 214 n. 887.

35 Hennes: Codex diplomaticus (cit. nota 34) vol. 1 p. 31; Pettenegg: Die Urkunden (cit. nota 34) pp. 2-3 n. 6 (datazione errata); Huillard-BréHolles (cit. nota 34) vol. $1 / 2$ pp. 510 sg.; RI 5/1 p. 218 n. 910.

36 Originale in Archivio di Stato di Palermo, Tabulario della Magione, 21, copie in Archivio di Stato di Palermo, Miscellanea Archivistica, II, 22, f. 55r-55v, Archivio Segreto Vaticano, Reg. Vat. 9, ff. 233v-234r, regesto in Petrus Pressuttr : Regesta Honorii papae III, Roma 1888, vol. 1, p. 195 n. 1172.

37 Huillard-BréHolles (cit. nota 34) vol. 1/2 p. 673-674, regesto in: RI 5/1 p. 242 n. 1049. Si veda anche Giuseppe Simoni: Cronistoria del Comune di Medicina, Bologna 1880, pp. 78sg.; Kurt Forstreuter: Der Deutsche Orden am Mittelmeer, Bonn 1967 (Quellen und Studien zur Geschichte des Deutschen Ordens 2), p. 147; Mario Fanti/ Giancarlo Roversi: S. Maria degli Alemanni in Bologna, Bologna 1969, p. 17. 
Hubert Houben, Dilectis filiis magistro, concede ai Teutonici il diritto di prendere le armi per difendere i castelli lasciati sotto la loro custodia da Federico II anche contro i cristiani ${ }^{38}$. I Teutonici che, in realtà, furono impegnati in azioni militari solo a partire dal 1210 incirca $^{39}$, avevano già combattuto contro gli Arabi insorti in Sicilia nel $1219^{40}$, giocando quindi ormai un ruolo di esperti di guerra e di tecniche di fortificazione, esattamente come i Templari e gli Ospitalieri, anche nei territori italiani.

I Teutonici furono anche i primi frati di un ordine religioso-militare ad ottenere dalla Sede Apostolica una concessione diretta di beni a Roma: nel 1220, Onorio III diede loro la chiesa di Santa Maria in Domnica al Celio che rimase per quasi un secolo la Sede romana dell'Ordine. Tuttavia, la chiesa, una delle principali arcidiaconie della città, non fu data ai Teutonici a titolo definitivo, bensì in concessione temporanea che fu revocata nel 1310 da Clemente $\mathrm{V}^{41}$.

Il conflitto tra Federico II e la Chiesa romana non determinò mai effetti davvero negativi sugli ordini religioso-militari della Penisola: per quanto riguarda il Regno di Sicilia, la problematica delle perdite subite dagli ordini a seguito dello scontro fra $\mathrm{i}$ due poteri è stata molto ridimensionata dalla storiografia recente ${ }^{42}$; mentre, dal canto suo, il papato non operò mai distinzioni tra gli ordini in base alla loro lealtà verso la Chiesa o l'imperatore. Ovviamente, le missioni della difesa della Terra Santa o dell'evangelizzazione dell'Europa nord-orientale prevalsero sul contesto locale della Penisola.

Abbiamo ricordato il ruolo dei frati degli ordini religioso-militari come custodi dei castelli nel Regno di Sicilia all'epoca di Federico II. Nel 1265, con l'avvento di Carlo I d'Angiò, a causa della speciale predilezione del sovrano per gli ordini militari, gli esponenti dei Templari, degli Ospitalieri e, talvolta, anche dei Teutonici svolsero nel Regno delle mansioni di ufficiali regi in ambiti legati alle attività militari, la tesoreria e la navigazione, ma anche in altri settori, come la gestione delle miniere ${ }^{43}$. Negli stessi anni, anche la Sede Apostolica cominciò

38 Houben: Friedrich II. (cit. nota 7).

39 Marie Luise Favreau-Lilie: L'Ordine Teutonico in Terrasanta (1198-1291), in: Houben: L'Ordine Teutonico nel Mediterraneo (cit. nota 32) pp. 53-72, qui pp. 5960.

40 Kristjan Toomaspoeg: Les Teutoniques en Sicile (1197-1492), Roma 2003 (Collection de l'École française de Rome 321), p. 49.

41 Kristjan Toomaspoeg: Die Deutschordenskirche Santa Maria in Domnica im Licht eines unbekannten Inventars von 1285, in: QFIAB 83 (2003) pp. 83-101.

42 Si veda Toomaspoeg: Templari (cit. nota 19) pp. 65-67.

43 Sull'argomento degli ordini militari a servizio dei poteri laici ed ecclesiastici, si veda Henri Bresc: Gli Ordini ospedalieri e militari nel Mediterraneo, in: I Teutonici tra Sicilia e Mediterraneo. Atti del Convegno Internazionale (Agrigento, 24-25 marzo 2006), a cura di Antonino Giuffrida/Hubert Houben/Kristjan Toomaspoeg: Galatina 
a servirsi di frati degli ordini militari, soprattutto nella loro veste di difensori armati.

É abbastanza difficile datare la comparsa dei primi cubiculari, ossia le guardie del corpo del papa, templari e ospitalieri. Il circondarsi di membri degli ordini religioso-militari diventa un'abitudine dei papi più o meno all'epoca della conquista angioina della Sicilia: da una parte, deve essere considerato il risultato di un preciso disegno politico dei capitoli degli ordini, che volevano essere presenti presso tutte le corti europee, compresa la Curia romana, dall'altra, i decenni precedenti alla caduta di Acri accentuarono il ruolo dei cavalieri e dei preti degli ordini quali difensori della Terra Santa, ben accolti e influenti in Europa.

I Teutonici, benché non facessero parte degli ordini più rappresentati presso la Curia, trovarono il modo per accrescere la loro influenza, affidando l'incarico di procuratore generale dell'Ordine presso il papa ad un importante notaio apostolico, Giovanni da Capua ${ }^{44}$. Questi, membro della cancelleria pontificia dal 1230, in seguito divenne un frate dell'Ordine Teutonico, fatto piuttosto raro trattandosi di un nativo della Penisola, e dal 1257 viene citato come procuratore dell'Ordine.

L'adesione di Giovanni da Capua sembra essere stato un successo diplomatico per l'Ordine, come fanno supporre i risultati concreti delle sue attività. Così, papa Alessandro IV (1254-1261) fu uno dei pontefici più favorevoli ai Teutonici, sia in generale che rispetto alle singole Case ${ }^{45}$, e proprio nel caso dei Teutonici si manifestò per la prima volta la nuova politica pontificia di concedere agli ordini religioso-militari grandi proprietà monastiche abbandonate $o$ in stato di degrado.

Infatti tra il novembre del 1260 e il gennaio dell'anno successivo, Alessandro IV affidò ai Teutonici la canonica di San Leonardo di Siponto con il suo immenso patrimonio ubicato in Puglia e in Basilicata ${ }^{46}$. Questa concessione ebbe una duplice motivazione: da una parte, essa risale a pochi mesi dopo la cocente sconfitta dell'Ordine contro i Lituani nella battaglia di Durbe cosicché avrebbe dovuto dare un nuovo sostegno economico e morale ai Teutonici;

2007 (Acta Theutonica 4), pp. 17-46. Sui membri degli ordini militari a servizio della corte angioina, Toomaspoeg: Templari (cit. nota 19) pp. 70-72.

44 Jan-Erik Beuttel: Der Generalprokurator des Deutschen Ordens an der römischen Kurie. Amt, Funktionen, personelles Umfeld und Finanzierung, Marburg 1999 (Quellen und Studien zur Geschichte des Deutschen Ordens 55); Fulvio Delle Donne: Giovanni da Capua, in: DBI 55 (2000) pp. 759-761.

45 Ad esempio, i numerosi atti emessi a favore della Casa padovana dell'Ordine cf. Toomaspoeg: La fondazione (cit. nota 6).

46 Regesto di S. Leonardo di Siponto, a cura di Fortunato Camobreco, Roma 1913 (Regesta Chartarum Italiae 10), p. 130 n. 195, p. 132 n. 197. 
dall'altra, la canonica, situata vicino ad uno dei più importanti assi viari del Mezzogiorno, era diventata da tempo una spelunca latronum ${ }^{47}$.

In realtà, non fu certo questa la prima volta che un ordine religioso-militare si era impossessato di un'istituzione ecclesiastica italiana tanto che casi di compravendite, acquisizioni per donazione e anche usurpazioni di chiese e monasteri da parte dei Templari, Ospitalieri e Teutonici sono frequenti in tutta la Penisola. Il possesso a lungo termine di queste chiese, come la Santissima Trinità dei Teutonici a Palermo, acquisita manu militari, o Santa Maria dell'Aventino dei Templari, ricevuta in donazione da parte dei Benedettini cluniacensi ${ }^{48}$, implicava sempre una esplicita o tacita approvazione da parte del papato. Tuttavia, la concessione diretta di un monastero o di un qualunque bene ecclesiastico da parte della Sede Apostolica ad un ordine militare costituì senza dubbio una rarità almeno sino al 1260 .

Un'altro esempio della politica pontificia di concessione di monasteri ci viene dal pontificato di Clemente IV, seppure secondo modalità diverse, dal momento che il bene in questione, il priorato benedettino di Santa Eufemia in Calabria, fu semplicemente occupato dagli Ospitalieri, azione in seguito approvata dal papa ${ }^{49}$.

Comunque, gli esempi più rilevanti di concessioni pontificie provengono da un'epoca più tardiva, successiva alla caduta di Acri, nel corso degli ultimi tentativi di espansione militare verso Oriente.

Il 22 settembre del 1297, con le lettere apostoliche Dum paterne considerationis $^{50}$ e Inter cetera mundi loca ${ }^{51}$, Bonifacio VIII diede agli Ospitalieri, principale ordine militare presente nel Regno di Sicilia, due importanti monasteri benedettini nel Mezzogiorno continentale, Sant'Angelo del Palazzo, presso Acquaviva Colle di Croce, e la Santissima Trinità di Venosa. Le due concessioni furono effettuate secondo modalità lievemente diverse: Sant'Angelo era un monastero che soffriva da tempo per l'incuria e la povertà della comunità, al punto di essere temporaliter et spiritualiter collapsum, cosicché i monaci l'avevano già consegnato, con il benestare del vescovo locale, agli Ospitalieri. Il cenobio venusino era invece direttamente soggetto alla Sede Apostolica e il papa motivò la sua concessione da una parte con le meritevoli attività dei Giovanniti, intrepidi pugiles, e, dall'altra, con l'incuria e la cattiva amministrazione in cui

47 Hubert Houben: „Iuxta stratam peregrinorum“: la canonica di S. Leonardo di Siponto (1127-1260), in: RSCI 56 (2002) pp. 323-348; San Leonardo di Siponto. Cella monastica, canonica, domus Theutonicorum. Atti del Convegno internazionale (Manfredonia, 18-19 marzo 2005), a cura di Hubert Houben, Galatina 2006 (Acta Theutonica 3).

48 Silvestrelli: Le chiese (cit. nota 26).

49 Salerno: Gli ospedalieri (cit. nota 18); Toomaspoeg: Templari (cit. nota 19) pp. 73sg.

50 Delaville Le Roulx: Cartulaire (cit. nota 13) vol. 3 pp. 721 sg. n. 4386.

51 Ibid. pp. 722 sg. n. 4387. 
versava il monastero. In entrambi i casi, i Benedettini rimasti potevano continuare a risiedere nei loro monasteri. Inoltre, nel gennaio dell'anno successivo, Bonifacio VIII fece annullare tutti i contratti di locazione con i beni della Santissima Trinità di Venosa stipulati in passato dalla comunità benedettina $^{52}$.

Sebbene esclusi dalla categoria dei beneficiari di speciali concessioni, i Templari godettero di un'altra forma di riconoscimento da parte del papato nell'ultimo quarto del XIII secolo, quando la Sede Apostolica mise sotto la loro custodia una serie di siti fortificati nel Patrimonium Beati Petri. Di norma si trattava di fortificazioni situate nell'area centrale e meridionale del Lazio e nell'Abruzzo, fra cui alcuni siti davvero prestigiosi, come il castrum di Cencelle (Tarquinia) o il castello di Santa Maria di Scurcola ${ }^{53}$, che non furono, contrariamente a quanto talvolta viene riportato, date in proprietà, bensì solo in custodia temporanea ai Templari. Certamente, anche la semplice custodia implicava l'usufrutto delle rendite di questi siti e procurò all'Ordine delle risorse notevoli.

3. Gli ordini militari in Italia dopo il Processo ai Templari

I Templari di stanza presso la Curia, cubiculari o castellani che fossero, vissero la loro stagione più felice sotto il pontificato di Bonifacio VIII, quindi solo pochi anni prima della soppressione dell'Ordine. Non vorrei entrare nell'argomento del processo ai Templari, peraltro oggetto di una sezione dell'ultimo convegno dei medievisti a Leeds, e vorrei solo fare alcune osservazioni sul suo impatto nel territorio italiano.

Le trasformazioni subite dagli ordini religioso-militari dopo la caduta di Acri non provocarono effetti immediati nella Penisola Italiana. Così, i grandi cambiamenti avvenuti all'interno dell'Ospedale di San Giovanni di Gerusalemme a seguito delle riforme di Guglielmo di Santo Stefano e il trasferimento del suo capitolo a Rodi, all'inizio del XIV secolo, o anche l'evoluzione simile, forse ancora più radicale, subita dall'Ospedale di Santa Maria dei Teutonici, non determinarono echi profondi in Italia e non si manifestarono nei rapporti degli ordini con il papato ${ }^{54}$.

Il caso dei Teutonici è emblematico: è vero che nel 1310, in un momento difficile della loro storia, trovandosi anch'essi sotto processo, Clemente V tolse

52 Pridem diligentius attendentes, ibid. pp. 729 sg. n. 4399.

53 Si veda Silvestrelli: Le chiese (cit. nota 26).

54 Si veda Anthony Luttrell: The Hospitallers and the Papacy, 1305-1314, Stuttgart 1998 (Forschungen zur Reichs-, Papst- und Landesgeschichte 2). 
loro la chiesa di Santa Maria in Domnica a Roma ${ }^{55}$ e si potrebbe pensare ad una politica repressiva della Sede Apostolica nei confronti dei possedimenti italiani dell'Ordine, se non fosse che il papa, allo stesso momento, intervenne in favore dei Teutonici nel corso della loro lite con il vescovo di Cefalù per il possesso della chiesa della Santissima Trinità di Polizzi in Sicilia ${ }^{56}$.

Il processo ai Templari ebbe invece un impatto diretto sul territorio della Penisola Italiana, dove fu celebrato con delle modalità particolari. Prima di tutto, in Italia non fu seguita una linea unitaria riguardo al destino dei Templari e in ogni singola regione, l'azione giudiziaria si svolse e si concluse in modi diversi. In alcune aree geografiche, come quelle direttamente soggette alla Curia $^{57}$ o il Regno angioino di Sicilia ${ }^{58}$, il processo implicò l'estorsione delle confessioni con la tortura e terminò con la condanna assoluta ed inappellabile degli accusati; in altre, come quelle sottoposte al giudizio del commissario pontificio Rinaldo da Concorezzo nell'Italia centro-settentrionale ${ }^{59}$, i Templari furono invece addirittura assolti; mentre in altre ancora, come la Sicilia aragonese, non vi fu un vero e proprio processo e i Templari rimasti furono fatti tacitamente confluire nelle fila degli Ospitalieri ${ }^{60}$.

Anche il destino dei possedimenti templari non fu univoco: come era stato predisposto dalla lettera apostolica Ad providam Christi del 2 maggio $1312^{61}$, per

55 Forstreuter: Der Deutsche Orden (cit. nota 37) p. 160; Toomaspoeg: Santa Maria in Domnica (cit. nota 41).

56 Una prima lettera del pontefice, Conquesti sunt nobis, fu indirizzata alle autorità ecclesiastiche palermitane nel luglio (1-7) del 1309, il papa invocò allora del diritto dei Teutonici di poter costruire delle chiese e cappelle, diritto che il vescovo di Cefalù non aveva rispettato quando aveva vietato la costruzione della chiesa teutonica di Polizzi, e ordinò di convocare una riunione per risolvere il contenzioso: la lettera è pubblicata in: Mongitore: Monumenta (cit. nota 33) p. 214, dall'originale nell'Archivio di Stato di Palermo, Tabulario della Magione, 496, regesto in: Toomaspoeg: Les Teutoniques (cit. nota 40) p. 729 n. 520 . Il 13 marzo del 1311, con la lettera Qua nobis, Clemente V indirizzò un simile ordine alle autorità ecclesiastiche di Monreale: la lettera, custodita in Tabulario della Magione, 516, è inedita, regesto in ibid. pp. 734sg. n. 535. In realtà, il diritto al quale si riferivano i Teutonici riguardava sì l'edificazione delle chiese e cappelle, ma solo nei territori appena conquistati dalle mani degli ,infedeli' e quindi la sua applicazione nel contesto siciliano indica la benevolenza del papa al riguardo dei Teutonici e, forse, anche una percezione della Sicilia aragonese come terra da ,riconquistare alla religione'.

57 Gilmour-Bryson: The Trial (cit. nota 26).

58 Giovanni Guerrieri: I Cavalieri Templari nel Regno di Sicilia, Trani 1909; Cristian Guzzo: Templari in Sicilia. La storia e le sue fonti tra Federico II e Roberto d'Angiò, Verbania 2003 (Insignia et Arma).

59 Come dimostra bene Elena Bellomo: The Templer Order (cit. nota 31) pp. 175-208.

60 Litterio Villari: Templari in Sicilia, Latina 1993; Kristjan Toomaspoeg: La fine dei Templari in Sicilia (1305-1326), in: Religiones Militares (cit. nota 30) pp. 155-170.

61 Johann Christian LüNIG: Codex Italiae Diplomaticus quo non solum multifariae Investiturarum Literae, ab Augustissimis Romanorum Imperatoribus Italiae Principibus 
lo più questi furono consegnati agli Ospitalieri, ma in molti casi il passaggio di proprietà fu disturbato o impedito dalle usurpazioni da parte di privati o dall'interferenza dei poteri pubblici, come quello angioino. Per quanto riguarda i territori direttamente sottoposti alla Sede Apostolica, l'Ospedale non ebbe difficoltà nel prendere possesso della case templari, compreso il complesso monumentale di Santa Maria all'Aventino, ma non ebbe alcun diritto sui siti che erano stati tenuti in custodia dal Tempio su incarico del papato ${ }^{62}$.

Successivamente, la Curia avignonese e romana del XIV secolo non esercitò sugli ordini religioso-militari la stessa influenza del secolo precedente, benché fosse rimasta il loro diretto superiore e uno dei loro principali interlocutori. Nel Trecento, i più importanti ordini militari rimasti nella Penisola Italiana, l'Ospedale di San Giovanni e quello di Santa Maria dei Teutonici, accrebbero il loro patrimonio e la loro potenza economica, ma le loro singole Case, malgrado i tentativi di controllarle, svolsero spesso per proprio conto attività politiche e diplomatiche in base al contesto in cui si trovarono. Inoltre, nel caso dei Giovanniti, le loro commende furono spesso gestite come semplici benefici ecclesiastici da commendatori locali, come ad esempio Roberto Diana in Sicilia ${ }^{63}$.

Il papato dovette riflettere sul ruolo degli ordini militari nel nuovo quadro politico occidentale dopo la perdita della Terra Santa e l'evangelizzazione definitiva dei confini occidentale e nord-orientale dell'Europa. Al centro di questa riflessione erano anche i possedimenti italiani degli ordini, in particolare del più ricco e potente di essi, l'Ospedale di San Giovanni.

I primi a voler conoscere l'effettivo stato dell'economia e del personale degli ordini militari in Italia furono gli stessi capitoli degli Ospitalieri e dei Teutonici che, a partire dal XIV secolo disposero che si realizzassero regolarmente delle visite nei loro distaccamenti nella Penisola e fecero stilare inventari dei beni posseduti. Un buon esempio è costituito dall'inventario dei beni del priorato giovannita di Roma, redatto nel 1334, contenuto nel «Liber Prioratus Urbis», recentemente pubblicato da Dominique Moullot ${ }^{64}$, che resta la fonte più dettagliata e precoce per la conoscenza del patrimonio dell'Ordine nell'Italia centrale.

$\&$ Proceribus concessae atque traditae; verum etiam alia insignia varii generis Diplomata, tam edita quam multa anecdota, ipsos concernentia continentur, Francoforte Meno/ Leipzig 1725-1735, vol. 2, col. 1651-1656; Regestum Clementis Papae V ex Vaticanis archetypis sanctissimi domini nostri Leonis XIII Pontificis Maximi iussu et munificentia nunc primum editum. Cura et studio monachorum Ordinis S. Benedicti, Roma 1887, anno VII, pp. 65-68 n. 7885; Lamattina: Regesta (cit. nota 8) pp. 248-252 n. 1106.

62 Gilmour-Bryson: The Trial (cit. nota 26); Silvestrelli: Le chiese (cit. nota 26).

63 Toomaspoeg: Templari (cit. nota 19) pp. 88-96.

64 Dominique Moullot: Le Liber Prioratus Urbis de l'Ordre de Saint-Jean-de-Jérusalem: édition critique du Vat. Lat. 10372, Bari 2004 (Gran Priorato di Napoli e Sicilia del Sovrano Militare Ordine di Malta, Melitensia 12). 
Non è un caso che l'inventario del 1334 sia finito in possesso della Curia pontificia (l'originale del manoscritto si trova oggi nella Biblioteca Vaticana) che mise in atto essa stessa dei tentativi al fine di conoscere l'entità dei beni degli Ospitalieri per capire se fossero stati in grado di combattere i Turchi nel Mediterraneo.

Il 10 febbraio del 1373, negli anni immediatamente precedenti al ritorno della Sede Apostolica a Roma, Gregorio XI, ancora ad Avignone, con la lettera Ex certis rationabilibus causis, ordinò un'inchiesta di vastissime dimensioni sul patrimonio europeo dell'Ospedale, compresi i suoi beni nella Penisola Italiana ${ }^{65}$. Come dichiarava il pontefice (nella sua missiva), l'inchiesta era destinata alla riforma dell'intero ordine, in spiritualibus ac temporalibus, per migliorare la difesa e la diffusione della fede cristiana. L'indagine doveva essere svolta dai vescovi locali, in ogni singola diocesi, e doveva fornire informazioni sullo stato del personale, dei beni e dei redditi delle commende giovannite locali. I verbali di queste inchieste diocesane costituiscono una delle fonti principali per la storia dell'Ospedale di San Giovanni e si trovano nell'Archivio Segreto Vaticano; essi sono stati pubblicati per la parte riguardante la Francia e alcuni altri territori ${ }^{66} \mathrm{e}$, di recente, anche per le parte rignardente il Mezzogiorno d'Italia ${ }^{67}$

L'inchiesta del 1373 fu percepita dagli stessi Ospitalieri come una grave intromissione nei loro affari interni. Si trattava di un procedimento finora sconosciuto in Italia, dal momento che i rappresentanti della Sede Apostolica non avevano mai avuto accesso ai possedimenti degli ordini religioso-militari per i quali la riservatezza e la separazione della vita conventuale dal mondo esterno erano alle basi della loro esistenza. Certo, i possedimenti templari erano stati inventariati, ma solo dopo il loro sequestro, nel corso del processo all'Ordine. Inoltre i Giovanniti temevano che i risultati dell'inchiesta fossero utilizzati per imporre ai loro possedimenti europei nuovi obblighi fiscali.

Di conseguenza, vi fu una certa resistenza da parte dell'Ospedale di San Giovanni che fece dell'inchiesta pontificia un esempio delle difficoltà incontrate dalla Sede Apostolica nell'esercitare un controllo sull'Ospedale stesso e sugli

65 Si veda Anthony LutTrell: Introduzione, in : L'enquête pontificale de 1373 sur l'ordre des Hospitaliers de Saint-Jean-de-Jérusalem, a cura di Jean GLÉnisson, vol. 1: L’enquête dans le prieuré de France, a cura di Anne-Marie Legras/Robert Favreau, Paris 1987.

66 Ibid.

67 Sull'inchiesta nel Mezzogiorno si vedano: Raffaele Iorio: L'inchiesta di papa Gregorio XI sugli Ospedagliere della diocesi di Trani, Taranto 1996 (Gran Priorato di Napoli e Sicilia del Sovrano Ordine di Malta, Melitensia 1; Salvatore Fodale: San Giovanni in Sicilia: l'inchiesta di Gregorio IX sull'ordine gerosolimitano, in: Società, istituzioni, spiritualità. Studi in onore di Cinzio Violante, Spoleto 1994 (Centro italiano di studi sull'alto medioevo, Collectanea 1) e, adesso, L'inchiesta pontificia del 1373 sugli Ospedalieri di San Giovanni di Gerusalemme nel Mezzogiorno d'Italia, a cura di Mariarosaria Salerno/Kristjan Toomaspoeg, Bari 2008 (Università degli Studi della Calabria, Corso di Laurea in Storia e Conversazione dei Beni Culturali, Itinerario 10). 
ordini religioso-militari in generale. I prelati incaricati dell'indagine fornirono al papa dei verbali che, quasi sempre, rispecchiavano la volontà degli Ospitalieri di diminuire l'entità del loro patrimonio, sostenuti in quest'azione dai loro buoni rapporti con le gerarchie ecclesiastiche locali. Così, ad esempio nell'ambito siciliano, i vescovi delle due diocesi principali dove si concentravano le commende dell'Ospedale, Messina e Catania, arrivarono addirittura a non rispondere al papa. Tra gli altri prelati, solo il vescovo di Cefalù, tradizionalmente in conflitto con gli ordini militari, fornì a Gregorio XI una descrizione più dettagliata del loro patrimonio ${ }^{68}$, ma anche in questo caso gli Ospitalieri riuscirono a sminuire il reale valore della loro ricchezza ${ }^{69}$.

Peraltro, proprio in ambito siciliano si manifesta con più chiarezza l'effetto dello scisma del 1378, dal momento che i priori locali dell'Ospedale di San Giovanni oscillarono sempre tra l'obbedienza romana ed avignonese, a seconda degli orientamenti delle dinastie al potere ${ }^{70}$.

\section{La trasformazione dei baliati teutonici in benefici ecclesiastici nel XV secolo}

L'Ospedale rappresentò sempre un avamposto della Cristianità nelle lotte contro i Turchi e i suoi diritti patrimoniali non furono mai seriamente minacciati dal papato, anche dopo il deludente risultato dell'inchiesta del 1373. Diverso fu invece il caso dei possedimenti locali dell'Ordine Teutonico, il che mi porta all'ultimo argomento che mi ero proposto di affrontare nel corso di questa comunicazione, dedicato alla trasformazione delle commende degli ordini religioso-militari in benefici ecclesiastici.

Prima della fine del XIV secolo, l'Ospedale di Santa Maria dei Teutonici non era particolarmente noto in Italia per le sua attività nell'Europa nordorientale e le sue commende nella Penisola beneficiarono del sostegno della popolazione locale - se prendiamo in considerazione, ad esempio, le motivazioni espresse da coloro che fecero donazioni a suo favore - per lo più grazie alle loro attività caritative e assistenziali ${ }^{71}$. Il contesto mutò fra la fine del XIV e

68 Archivio Segreto Vaticano, Instr. Misc., 2807; L'inchiesta (cit. nota 67) pp. 271-274.

69 FodAle: San Giovanni (cit. nota 67).

70 Si veda ID.: Scisma ecclesiastico e potere regio in Sicilia, I. Il duca di Montblanc e l'episcopato tra Roma e Avignone (1392-1396), Palermo 1979; ID.: Il clero siciliano tra ribellione e fedeltà ai Martini (1392-1398), Palermo 1983.

71 Sulle donazioni patrimoniali a favore dei Teutonici, Kristjan Toomaspoeg: Base économique de l'expansion des bourgs siciliens. Éxemple des possessions de l'Ordre Teutonique dans la zone Corleone-Vicari-Castronovo, 1220-1310, in: Actas del XVII Congreso de Historia de la Corona de Aragón, „El món urbà a la Corona d'Aragó del 1137 als decrets de Nova Planta", Barcelona-Lleida, 7-12 septiembre 2000, Barcelona 2003, vol. 1, pp. 595-604. 
l'inizio del XV secolo, quando nella nobiltà italiana, in alcuni casi direttamente partecipante alle spedizioni dell'Ordine in Lituania, si diffuse la nuova ideologia dei Teutonici, quali combattenti contro i pagani della Prussia e della Livonia ${ }^{72}$. Nel Quattrocento, l'Ordine Teutonico fu spesso chiamato dagli abitanti della Penisola come „'Ordine di Prussia“, e, per la prima volta nella loro storia, i distaccamenti italiani dei Teutonici furono percepiti come possedimenti di un'istituzione, straniera'.

Questi beni, amministrati da pochi frati dislocati nel territorio italiano, avevano un valore economico considerevole, espresso nelle elevate rendite annue dei baliati teutonici. Nel XV secolo, la Chiesa romana cambiò visibilmente atteggiamento nei confronti di questi distaccamenti, considerandoli non più la base logistica di una guerra combattuta altrove - dopo la conversione dei Lituani alla fine del XIV secolo i Teutonici avevano perso il loro ruolo di difensori della Fede contro i pagani -, bensì semplici benefici monetari. Dunque la Sede Apostolica, che in passato aveva espropriato istituzioni monastiche del Regno di Sicilia a favore degli ordini religioso-militari, applicava adesso la stessa politica ai possedimenti teutonici, ma a suo proprio beneficio.

Di conseguenza, nel Quattrocento i Teutonici persero i loro due più ricchi baliati italiani, la Puglia e la Sicilia ${ }^{73}$, mentre il terzo, la ,Lombardia', resisté sino alla Riforma protestante, venendo poi in parte incorporato nel baliato austriaco dell'Ordine e in parte diviso tra la Repubblica veneziana e alcune congregazioni religiose, soprattutto i Gesuiti ${ }^{74}$.

Le modalità dell'esproprio furono diverse: in Puglia, il commendatore regionale dei Teutonici, Stefan Grube, che era anche un alto prelato (arcivescovo di Riga), entrato in conflitto con l'Ordine, affidò il baliato alla Curia pontificia

72 Kristjan Toomaspoeg: La guerre baltique au regard des sociétés de l'Europe méditerranéenne à la fin du Moyen Âge, in: Regards croisés sur la guerre sainte. Guerre, religion et idéologie dans l'espace méditerranéen latin $\left(\mathrm{XI}^{\mathrm{e}}-\mathrm{XIII}{ }^{\mathrm{e}}\right.$ siècle). Actes du Colloque international tenu à la Casa de Velázquez (Madrid) du 11 au 13 avril 2005, a cura di Daniel Baloup/Philippe Josserand, Toulouse 2006, pp. 399-412. Dei nobili della Penisola Italiana che hanno combattuto in Prussia si conosce prima di tutti Raimondo del Balzo Orsini: Hubert Houben: Raimondo Del Balzo Orsini e l'Ordine Teutonico, in: L'Ordine Teutonico tra Mediterraneo e Baltico: incontri e scontri tra religioni, popoli e culture. Atti del convegno internazionale. Bari/Lecce/Brindisi, settembre 2006, Galatina 2008 (Acta Theutonica 5), pp. 195-217.

73 Sulla perdita di questi due baliati, Bruno Schumacher: Studien zur Geschichte der Deutschordensballeien Apulien und Sizilien, in: Altpreußische Forschungen 18 (1941) pp. 187-230, 19 (1942) pp. 1-25.

74 Karl Schellhass: Die Deutschordenskommende zu Padua und die Jesuiten. Ein Beitrag zur Geschichte des Deutschordens in den Jahren 1511-1575, in: QFIAB 7 (1904) pp. 91-120; Johann RaIner: Zur Geschichte des Deutschordenspriorates SS. Trinità in Venedig vornehmlich im 16. Jahrhundert, in: Acht Jahrhunderte Deutscher Orden in Einzeldarstellungen, a cura di Klemens Wieser, Bad Godesberg 1967 (Quellen und Studien zur Geschichte des Deutschen Ordens 1), pp. 357-370. 
di Sisto IV. In Sicilia, il caso fu molto più complesso, dal momento che un ruolo importante fu giocato anche dal cardinal protettore dell'Ordine presso la Curia, Rodrigo Borgia. In un primo tempo, il commendatore locale dei Teutonici, temendo di essere punito dai superiori per la sua condotta indecorosa, consegnò il baliato al Borgia che ottenne il 2 maggio del 1492 da Innocenzo VIII la conferma per godere dei redditi dei possedimenti teutonici ${ }^{75}$, prima di trasformarli in un beneficio ecclesiastico quando egli stesso divenne papa (Alessandro VI) e cederli al cardinale Federico di Sanseverino a titolo di ringraziamento per il suo voto nel conclave ${ }^{76}$. Alla fine, la Corte di Ferdinando il Cattolico, che in realtà era all'origine di tutta la vicenda ${ }^{77}$, comprò il baliato dal cardinale di Sanseverino per creare sulla sua base una riserva patrimoniale di beni ecclesiastici vacanti, nota più tardi come la Commenda della Magione ${ }^{78}$.

\section{Conclusioni}

Gli esempi che ho riportato illustrano le varie fasi della politica pontificia nei confronti degli ordini religioso-militari nella Penisola Italiana. L'arrivo degli ordini, prima in veste di congregazioni assistenziali (gli Ospitalieri), poi come ordini militari veri e propri (i Templari), fu possibile grazie al costante sostegno del papato, in particolar modo di pontefici come Innocenzo II e Alessandro III che, agendo da diretti ed unici superiori degli ordini, intervennero presso le gerarchie ecclesiastiche e i poteri laici locali perché consentissero l'insediamento di Case ospitaliere e templari lungo le principali vie di comunicazione che conducevano da Occidente all'Oriente. Questo fenomeno corrispose cronologicamente alla diffusione in Italia delle congregazioni assistenziali della Terra Santa e dei Cistercensi.

Con Innocenzo III e Onorio III, gli ordini militari furono a servizio delle ambizioni politiche del papato nella Penisola, come illustra bene il caso dell'Ordine Teutonico, autorizzato dalla Sede Apostolica a combattere (nel Regno di Sicilia) anche contro i cristiani. Peraltro, le attività degli ordini in Oriente gli procurarono la speciale protezione del papato che prevalse nelle vicende politiche locali della Penisola. Così, anche in contesti difficili, come nel Mezzogiorno alla fine del regno di Federico II, o durante i diversi conflitti

75 Ad personam tuam, Mongitore: Monumenta (cit. nota 33) pp. 145-147.

76 Toomaspoeg: Les Teutoniques (cit. nota 40) pp. 895-898 nn. 1058-1070.

77 Antonio de la Torre: Unas noticias de la Orden militar de Caballeros teutones, in: Gesammelte Aufsätze zur Kulturgeschichte Spaniens 13/3, Münster 1958, pp. 270-274.

78 Antonino Giuffrida: Il potere del segno. La transizione della Magione da baliato a commenda in: Giuffrida/Houben/Toomaspoeg: I Teutonici (cit. nota 43), pp. 159202. 
veneto-genovesi, non vi furono ripercussioni notevoli sul patrimonio degli ordini militari in Italia.

Nella seconda metà del XIII secolo, i distaccamenti italiani degli ordini furono chiaramente percepiti come una base economica della Terra Santa e il papato intensificò il suo sostegno agli ordini, concedendo ai Teutonici, indeboliti dopo la battaglia di Durbe nel 1260, la canonica di San Leonardo di Siponto e incorporando nell'Ospedale, dopo la caduta di Acri, i monasteri benedettini di Sant'Angelo del Palazzo e Santissima Trinità di Venosa. I membri degli ordini militari entrarono a servizio diretto della Santa Sede, come esperti di questioni militari, e gli ultimi papi del secolo furono attorniati da Templari, Ospitalieri e Teutonici.

Il periodo avignonese del papato si apre con il Processo ai Templari che in Italia presenta delle modalità assai diverse a seconda delle singole regioni e illustra la mancanza di un progetto unico sul destino dell'Ordine. Al tempo stesso, dopo la perdita della Terra Santa, i due rimanenti grandi ordini religiosomilitari dovettero giustificare la loro esistenza presso il papato. Gli Ospitalieri ripiegarono a Rodi per combattere contro i Turchi, impresa che gli assicurò per secoli il sostegno della Sede Apostolica e del mondo cristiano, mentre i Teutonici si riservarono il più effimero ruolo di difensore della Cristianità contro i pagani del Baltico.

Di conseguenza, il destino dei possedimenti italiani dei due ordini fu diverso. Gli Ospitalieri continuarono ad usare la Penisola come base logistica per le lotte combattute nel Mediterraneo, prima di trasferire, nel XVI secolo, il loro capitolo in un territorio allora, italiano', l'isola di Malta. Il papato fece alcuni tentativi per conoscere lo stato dell'economia e del personale dei distaccamenti italiani dell'Ospedale, ma non riuscì mai a ricevere informazioni precise a loro riguardo o ad intromettersi nella distribuzione delle risorse monetarie prodotte dall'Ordine in Italia. L'Ordine Teutonico invece, a causa del suo allontanamento geografico dalla Penisola Italiana, non fu in grado di mantenervi la sua presenza e, dopo la sconfitta inflittagli dai Lituani e dai Polacchi, i suoi possedimenti più rilevanti furono espropriati dalla Santa Sede.

In questa comunicazione ho tentato di illustrare tre aspetti diversi dei rapporti intercorsi tra il papato e gli ordini militari, ovvero il sostegno, la collaborazione e l'interferenza. L'atteggiamento della Santa Sede dipese dal ruolo che si attribuiva ai possedimenti degli ordini militari in ogni singola epoca: luoghi di cura e di assistenza dei pellegrini e di raduno dei crociati, avamposti militari a servizio degli interessi del papato o centri di produzione di risorse economiche. Il caso italiano è interessante, sia per il grande impatto esercitato dagli ordini militari sul territorio, sia per la presenza della stessa Santa Sede nella Penisola e la sua vicinanza geografica con gli insediamenti degli ordini. 
Le lettere apostoliche relative agli ordini religioso-militari raccolte da Paul Fridolin Kehr e dai suoi collaboratori riguardano quindi un aspetto importante e caratteristico della storia medievale d'Italia.

\section{Zusammenfassung}

Die neudurchgesehenen Akten der Ritterorden waren eine wichtige Quelle im Editionsunterfangen der Italia Pontificia. Für die Mitarbeitern von Paul Fridolin Kehr waren sie ein Mittel, um viele unedierte Dokumente zu diesem Thema aufzuspüren.

Bringt man die Unterlagen der Italia Pontificia mit jenen zusammen, die bisher zwar unbekannt waren, aber in einschlägigen Studien publiziert oder aufgelistet sind, kann man versuchen, den logischen Weg der direkten Beziehungen zwischen den drei großen Militärorden, das heißt den Templern, den Johannitern und dem Deutschen Orden, und dem apostolischen Stuhl innerhalb der italienischen Halbinsel nachzuvollziehen.

Durch die Neulektüre einer Reihe von päpstlichen Quellen, die aus vier verschiedenen Jahrhunderten stammen, kann man die Entwicklung der Haltung nachvollziehen, die die Päpste bezüglich der Militärorden einnahmen, ausgedrückt in vier Paragraphen, die jeweils betreffen: - die Regel, die das Papsttum zur Gründung der Templer und Johanniter auf der Halbinsel im 12. Jahrhundert erlassen hat; - die Beziehung zwischen dem apostolischen Stuhl, den Templern, den Johannitern und dem Deutschen Orden im 13. Jahrhundert; das Schilderung zum Prozess über die Templer und die Schwächung des Papsttums im 14. Jahrhundert; - die Umgestaltung der Besitztümer des Deutschen Ordens in kirchliche Güter, die der päpstlichen Macht des 15. Jahrhunderts unterlagen.

Zum Schluss ist zu unterstreichen, dass die apostolischen Briefe für die Militärorden, gesammelt von Paul Fridolin Kehr und seinen Mitarbeitern, die einen wichtigen und charakteristischen Aspekt des mittelalterlichen Italiens betreffen. 